In the Melbourne experience (Harvey et al. 1993), ictal SPECT lateralized lesions and correlated with EEG and MRI findings; it was superior to ictal EEG in localizing value in some. (PPN II, 1994;p79).

Mesial temporal spikes interfere with working memory in a report of 8 adult patients with depth electrodes implanted to evaluate intractable epilepsy, at Johns Hopkins Epilepsy Center, Baltimore, MD. (Krauss GL, Summerfield M, Brandt J, Breiter S, Ruchkin D. Neurology Oct 1997;49:975980). The greatest impairment of spatial and verbal recall coincided with left hippocampal spiking.

\title{
FIRST TONIC-CLONIC SEIZURE: TREATMENT AND PROGNOSIS
}

The outcome of immediate treatment (carbamazepine, phenytoin, phenobarbital, or sodium valproate) or delayed treatment after a second seizure, in two groups of patients followed for a minimum of 3 years, was evaluated in a multicenter, randomized, open trial reported by the "FIRST Group" from Segrate, Italy. Patients aged 2 years and older were included, 27\% $<16$ years, and $67 \% 16-60$ years. Only $52(24 \%)$ of 215 patients assigned to immediate treatment of the first tonic-clonic seizure had a recurrence during follow-up, whereas $85(42 \%)$ of 204 randomized to delayed treatment experienced a relapse. Remote etiological factors, a secondarily generalized seizure, and epileptiform EEG abnormalities increased the risk and frequency of relapse. Young to middle-age adults, and patients treated acutely with benzodiazepines had a lower risk of relapse. The probability of a 1 year remission was $20 \%$ greater in the immediate treatment group compared to those beginning treatment only after a relapse, but the difference was not significant. Patients treated after the first or second seizure had the same probability of remaining seizure-free for 1 or 2 years. (Musicco M, Beghi E, Solari A, Viani F, for the Rirst Seizure Trial Group (FIRST Group). Treatment of first tonic-clonic seizure does not improve the prognosis of epilepsy. Neurology Oct 1997;49:991-998). (Reprints: Dr Massimo Musicco, Istituto di Tecnologie Biomediche Avanzate, Consiglio Nazionale delle Ricerche, Via Filli Cervi 93, 20090 Segrate (MI), Italy).

COMMENT. The risk of seizure recurrence after a first unprovoked seizure is reduced $50 \%$ by immediate initiation of antiepileptic therapy. The lessening of patient and parent anxiety and the avoidance of possible complications of a second seizure by early treatment must be weighed against the risks of adverse effects of medications. Since more than 50\% of untreated patients are seizure-free at the end of a 1 to 2 year follow-up period, many authorities elect to defer therapy pending a seizure recurrence. To prevent the unnecessary AED exposure of patients with a low recurrence risk, an individually determined therapeutic approach is most appropriate, having regard to risk factors such as age, etiological factors, and EEG findings. Reliance on the use of rectal diazepam to limit severity and duration of acute seizure recurrence should favor the delayed therapy approach.

A previous prospective study and report from Montefiore Medical Center, New York, involved 407 children followed for a mean of 6 years. The risk of seizure recurrence after a first unprovoked seizure was $37 \%$ at 2 years and $42 \%$ at 5 years. The authors emphasize the improved quality of life as justification for rational therapy and seizure control. (Shinnar et al. 1996; see Progress in Pediatric Neurology III, 1997;pp88-89). 